\title{
Design of an Atlas of Significant Natural Disasters
}

\author{
Denitsa Siteva $^{a}$, Silvia Marinova ${ }^{\mathrm{b}, *}$ \\ ${ }^{a}$ Eng. Denitsa Siteva \\ University of Architecture, Civil Engineering and Geodesy \\ 1, Hristo Smirnenski Blvd., 1164 Sofia, Bulgaria \\ e-mail: d.siteva@gmail.com \\ ${ }^{b}$ Eng. Silvia Marinova, PhD \\ University of Architecture, Civil Engineering and Geodesy; Laboratory on Cartography \\ 1, Hristo Smirnenski Blvd, 1164 Sofia, Bulgaria \\ e-mail: marinova_silvia@abv.bg \\ * Corresponding author
}

\begin{abstract}
Natural disasters change every year, which leads to anomalies and subsequently causing more and more damage. Therefore, it is important that people receive as much information for disasters as possible, in order to be aware of their power and consequences. The clearest way to transmit information about a disaster, before, during and after its occurrence, is to map it. This paper discusses the applicability of atlas mapping, and the process of creating an "Atlas of significant natural disasters, around the world, for 2019". The Atlas collects, and consolidates historical and up-to-date information on natural disasters around the globe, including tropical cyclones, earthquakes, floods, pandemics and wild fires. By combining this information with data on population and infrastructure, the Atlas provides users with a better understanding of current hazards and their potential impacts.
\end{abstract}

Keywords: atlas, natural disasters, disaster mapping, atlas design

\section{Introduction}

Natural disasters are part of the life on earth. Some big disasters, or how they are called in this work, significant, can leave consequences for which will be needed years to restore. Humans are unable to prevent disasters especially natural disasters but assessment and planning can mitigate the various harmful effects and damage of such events to much extent. International agencies and governments of vulnerable countries are making a transition towards disaster management. Disaster Management is one of the most promising research fields because of its important economic and social implications. A lot of research has been done on Disaster Management for early warning and timely intervention to reduce the disruption and loss of life (Kaur et al., 2019).

Disaster management is directly related to disaster mapping. Disaster mapping is a tool for assessing storing and conveying information on the geographical location of a disaster occurrence and spread of the effects or probable effects of disasters. When disasters are mapped, the information for their intensity, the area/region of their occurrences and their impact must be assessed, so as to have information/data about the damages caused by them to the area/population specific or probable damages or impact likely to be caused. Proper mapping is helpful not only for disaster preparedness but also in rescue and relief operations with greater accuracy and speed. The significant role of cartography and maps in disaster management is presented in the book Successful Response Starts with a Map (National Research Council, 2007).

In 2019, 396 natural disasters were recorded in EM-DAT with 11,755 deaths, 95 million people affected and 103 billion US\$ in economic losses across the world (Natural Disasters 2019, CRED, 2020). And how many of the affected people had access to information about natural disasters happening at their location, in addition to what was reported on the news? Often the maps that are created during and after the disasters, are made difficult to read, and so people are left without the ability to understand and use this important information. Therefore, the purpose of the "Atlas of significant natural disasters, around the world, for 2019" is to provide easily accessible and understandable information on significant natural disasters for 2019, in this case it is a collection of disasters worldwide, but it can easily be created as well of disasters for a specific place, country or continent. It was chosen for the atlas to present the maps of 7 natural disasters that occurred in 2019, which left a big mark behind, and most of them can easily be called anomalies. Each map is accompanied by an information page that contains images, scientific and statistical data. The atlas is designed so that made to be easy to read by a large group of people, no matter the age, education or profession of the users.

And the goal is for people to get more information and knowledge about disasters, not to underestimate their power, and to be more and more prepared for the next ones to come. So that human casualties and economic losses can be reduced. 


\section{The Role of Atlas Mapping in Disaster Risk Reduction}

In the hours and days following the disaster, humanitarian organisations such as the Red Cross are looking for answers to several key questions: Where were the people during the disaster? How many people are affected? What are their basic needs? Coordinating emergency response and providing assistance to victims as quickly as possible, is crucial, and important to minimizing damage. In this case, is used the term rapid mapping. This term is used to notate the quick recording of information on a disaster. Such mapping is performed immediately or as soon as possible after the disaster happens in case of humanitarian crises, natural disasters, and man-made emergency situations. The damage assessment maps should be available within 24 hours and updated daily, and the situation maps and forecasts of the evolution of situations should be ready within the first few days or weeks after a crisis (as recommended by GMES) (Three-dimensional maps for disaster management, 2012). In disaster management, five main types of cartographic products can be distinguished: risk maps, early warning maps, reference maps, assessment maps, and thematic maps. Each of these maps is prepared at a different stage in dealing with the disaster and is of interest to various institutions dealing with the damages. Each of them is differently detailed and presents the information in a different way. For example, map that is used for immediate and urgent response does not have the same completeness and content as a map for impact assessment.

Disaster mapping includes mapping of the affected areas, which must be distinguished and shown on the map. This can be done by using ground-based observations or by remote monitoring devices, such as aerial photographs or satellite imagery. The importance of spatial information for emergency response and disaster risk reduction is described by Zlatanova and Li (2008), Konecny et al. (2010).

By providing information from satellites, it is possible to process and make the necessary maps from anywhere in the world. This allows people who are not affected by the disaster to provide support to the affected area. This also means that the equipment needed to create the maps will continue to function, even if all of the infrastructure is damaged or destroyed in the disaster area. Unfortunately, there are also some drawbacks to using data remotely. The availability of data is not always present or takes a long time to acquire the image, and many times satellite imagery is non-functional due to a blurred sky in most cases caused by the disaster.

The use of ground surveillance involves the collection and provision of georeferenced data from the exact place of the disaster. Unfortunately, this is also a relatively slow process for finding data for the making of cartographic products. It is important that disaster map information is freely available to anyone who wants to receive it or provide more data that can help the process, completeness and provide assistance. An example of an open source mapping platform is OpenStreetMap, which has been used to help create maps for a number of natural disasters. But there are still many places that has lack of detailed maps.

Atlas mapping is used in the creation of new updated and more detailed groups of maps of regions at risk of natural disasters, parts of the world or communities for which there is no mapped information that is easily accessible and stored in one place. Atlas mapping can be used in various directions:

- Tracing the trajectory and development of the disaster, collecting all the rapid maps created at the various stages of the disaster.

- When an area is often affected by a particular type of natural disaster, the creation of an atlas, collecting all maps of previous natural disasters, helps to identify the places through which disasters often pass. That can help selecting locations for building health zones and disaster response centres.

- Atlases are also created to illustrate the assessment of damage from a disaster or to summarise the impact of natural forces for a given season, year or period of time on a small area, country or continent.

- It is no coincidence that atlases are used in schools, they present the information much more clearly and extensively, because they have the possibility to add many fields and pages with text and illustrations.

- Collecting many maps in one place (atlas), immediately increases the amount of information that will be transmitted, the more it is contained in one atlas the more the user will receive only in the presence of one cartographic creation.

Disaster mapping systematizes, concretizes, illustrates and transmits very accurately the information needed by the user. By using more than one map to present a natural disaster, it makes it possible to convey much more detailed information.

\section{Significant Natural Disasters in 2019}

\subsection{Data source and classification}

The "Atlas of significant natural disasters, around the world, for 2019" collects and transmits data from trusted sources of cartographic and statistical general world information such as: The World Health Organization (WHO), EM-DAT, The International Disaster Database, International Federation of Red Cross and Red Crescent Societies (IFRC), Munich Re's, NatCatSERVICE, Statista, United Nations Office for Disaster Risk Reduction( UNISDR), United Nations Office for the Coordination of Humanitarian Affairs (OCHA). For each of the disasters, sources of thematic information were used, and in fact sites, articles and statistics specifically for the disaster, the territory through which it passed and the people affected, such as: Médecins Sans Frontières (MSF) emergency response to Cyclone Idai and flooding, Track The Tropics, The Bushfire and Natural Hazard 
Cooperative Research Centre (BNHCRC), USGS National Earthquake Information Center (NEHRP), M 8.0 - 78km SE of Lagunas, Peru.

A single scale for disaster general classification has not yet been adopted for the whole world to use. The very basic classification of disasters is based on whether the event is natural or man-made. Natural disasters can be grouped based on the origin namely: biological, geophysical, hydrological, climatological and extraterrestrial events. Further classification into main type, sub-type, and subsub type divides broad disaster groups to specific disaster types. And this is based on one of the most popular classifications for disasters made by EM-DAT (2021). This is the classification used to distinguish the five types of natural disasters, selected as significant, that are presented in the "Atlas of significant natural disasters, around the world, for 2019":

a) Meteorological:

Tropical Cyclone Idai "The third deadliest tropical cyclone in the Southern Hemisphere";

Hurricane Dorian "The most powerful hurricane recorded in the open region of the Atlantic Ocean, outside the Caribbean Sea";

b) Hydrological:

Floods from monsoon rains in India "The monsoon season of 2019 has the highest recorded rainfall in a quarter of a decade";

c) Climatological:

Wild fires in Australia "The fire season 2019-2020 in Australia is considered the worst in the history of the New South Wales Fire Service";

d) Geophysical:

Earthquakes in Peru and Abania "The magnitude 8Mw earthquake in Peru has been declared the strongest for 2019" and "A magnitude 6Mw earthquake in Albania has been declared the deadliest for 2019";

e) Biological:

Epidemic of Ebola in the Democratic Republic of the Congo "The second largest recorded Ebola epidemic".

These six natural disasters are picked, after studying and comparing a number of statistics and databases. They are called significant natural disasters because they have caused the worst consequences, such as the largest number of people affected, the largest territorial scope, the largest damage. Most of them top the statistics on devastating natural disasters, surpassing previous records of previous disasters of the same kind.

\section{Design of an Atlas of Significant Natural Disasters in 2019}

When creating the "Atlas of significant natural disasters, around the world, for 2019" and choosing the design, the main purpose of it was to be easy to read, and to be understood even by non-professional users.

The first two pages present the content and general information about the purpose of atlas mapping and the benefits of disaster mapping. On the next pages two world maps are presented. The first one shows the location of all natural disasters for 2019, and according to the classification, the five types of natural disasters are marked with dots in different colours (Figure 1.).

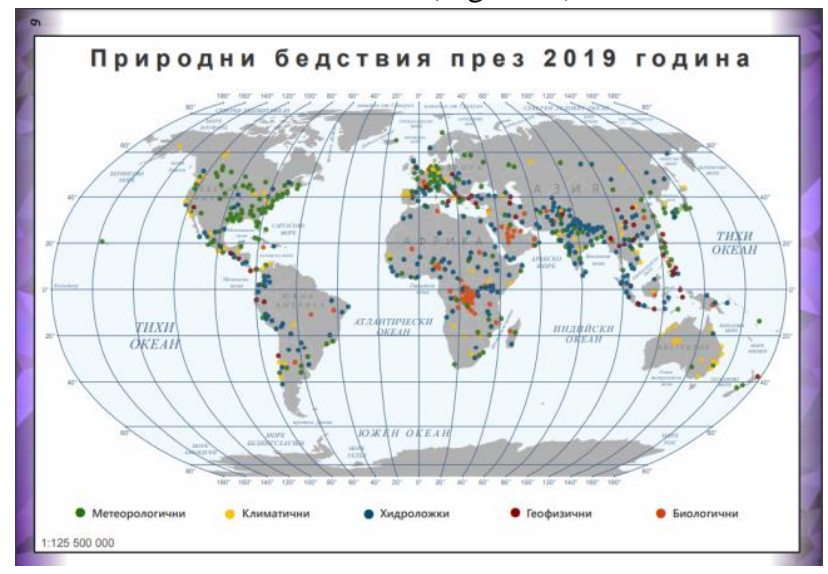

Figure 1. Map "Natural disasters in 2019".

The next world map shows the locations of the seven natural disasters that have been selected as significant for 2019 (Figure 2.), for each of them individual maps are presented in the following pages of the atlas. Again, the different types of natural disasters are represented by separate symbols, which, however, are much more graphic and visual. At first glance the user can easily recognize which symbol for which type of natural disaster refers to.

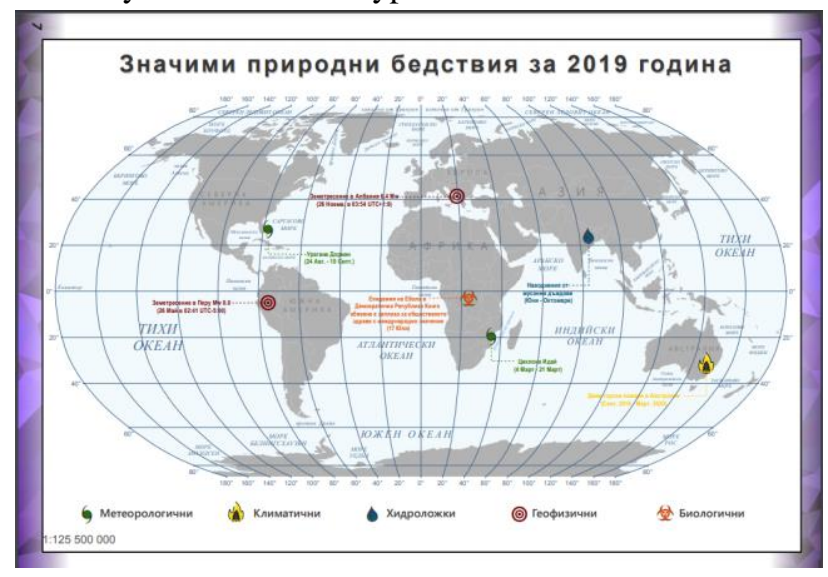

Figure 2. Map "Significant natural disasters for 2019".

After the two world maps are placed the separate maps for each disaster, and the accompanying them pages with additional information.

\subsection{Design of the pages with maps}

During the creation of the maps, attention was paid to the selection and design of the cartographic symbols and colours. In order to convey the most information, point, line and area symbols are made and used. For example, in the cyclone Idai's map, area type symbols indicate the strength of the wind and the affected areas, and line type symbols show the trajectory of the cyclone, and separate the different countries and provinces (Figure 3.). 


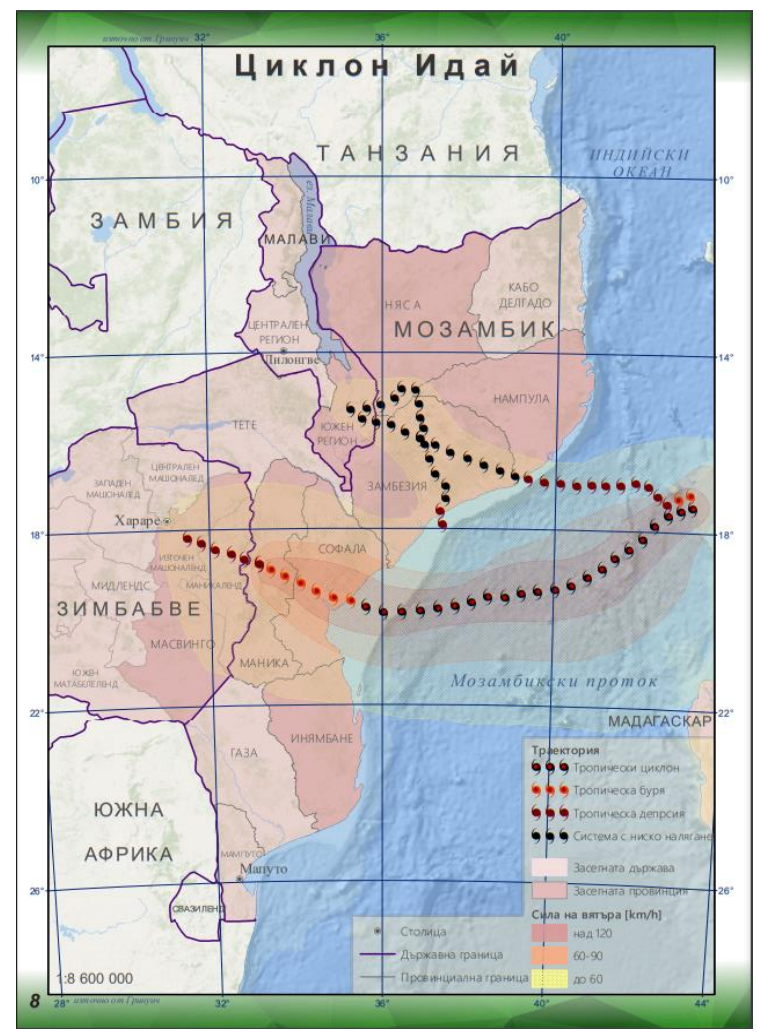

Figure 3. Map "Cyclone Idai".

The same methods were used in the map for hurricane Dorian, but because the mapped disasters are not just meteorological, other symbols of the same types, have been created for the following maps.

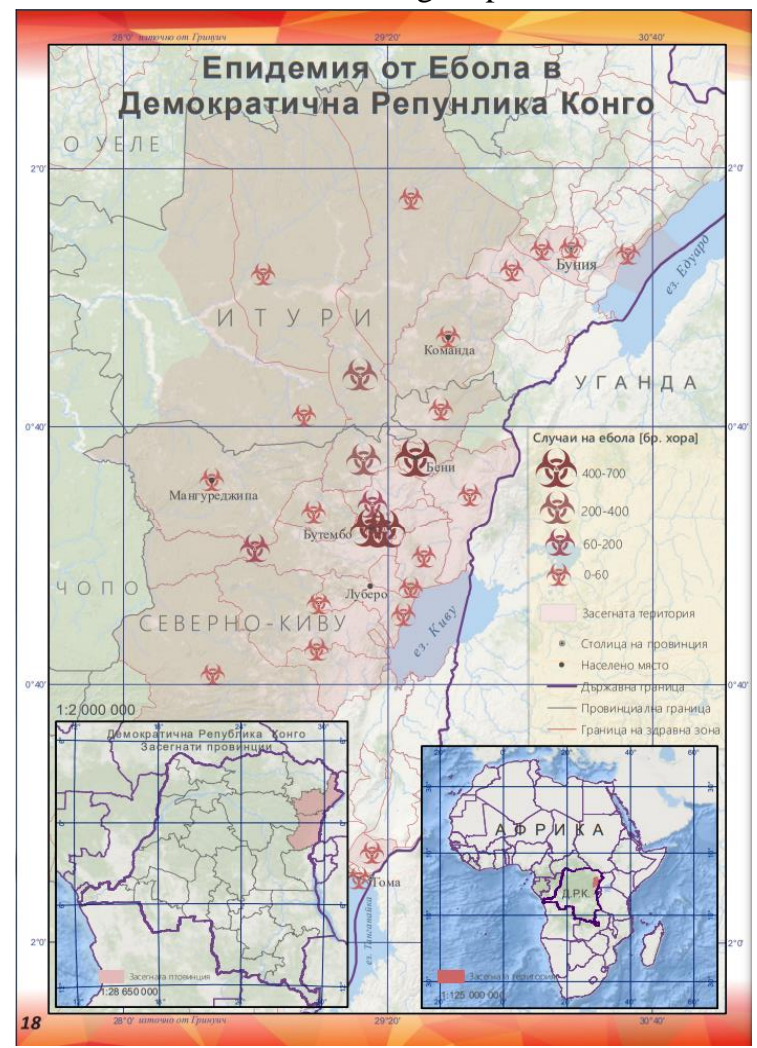

Figure 4. Map "Ebola epidemic in the Democratic Republic of Congo".
As another example, is the map of the Ebola epidemic in the Democratic Republic of Congo (Figure 4.), it must be said that the insert map method is used here, which is applied not only in this map but also in the map of significant earthquakes (Figure 5.), in order to be able to show much more specifically the location of the disaster.

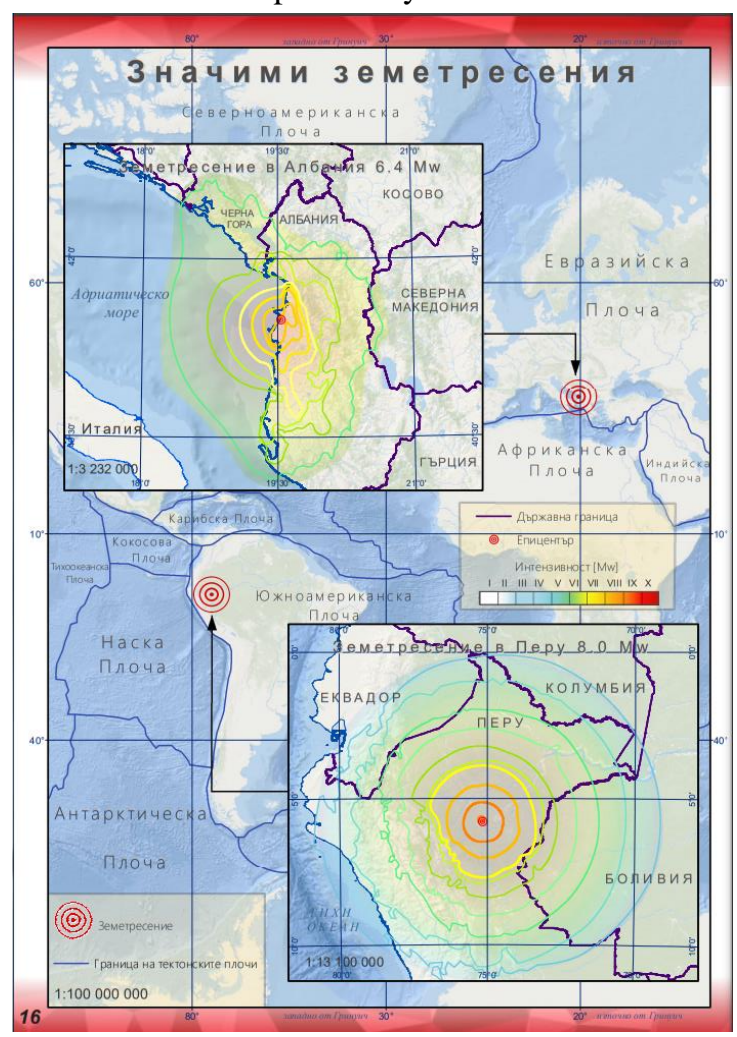

Figure 5. Map "Significant earthquakes".

The choice of colour palette is also well thought out. Each page has a frame, the colour on which it is based on the colours that were used to indicate the different types of natural disasters in the first world map (Figure 6.).

This design decision has been taken again for the convenience of the user. The atlas is designed for printing, that's why the colours are made CMYK colour model.

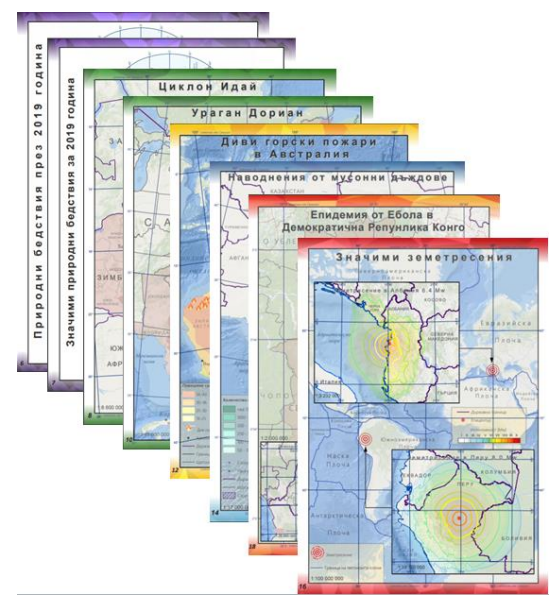

Figure 6. Colour choice. 


\subsection{Design of the pages whit additional information.}

The additional sheets of information are one of the parts that distinguishes the "Atlas of significant natural disasters, around the world, for 2019". With them it is certain that the user, in addition to cartographic information, will also receive visual, statistics and scientific information. Each component placed on the pages is relevant and carries separate information. At the top of the page several features are presented (Figure 7):

- In the middle, the title;

- In the right corner, next to the title, is placed an image of the globe, on which the continent on which the disaster occurred, is coloured, and the location of the disaster is indicated with a symbol (hornbeam);

- Below this is a scientific description of the disaster, accompanied by an image;

- Then there is a text box entitled "history of the disaster", it describes the development of the disaster from its first day of occurrence to the last, with dates, affected areas and consequences;

- And behind the text for background, is a graphic image, describing the disaster.

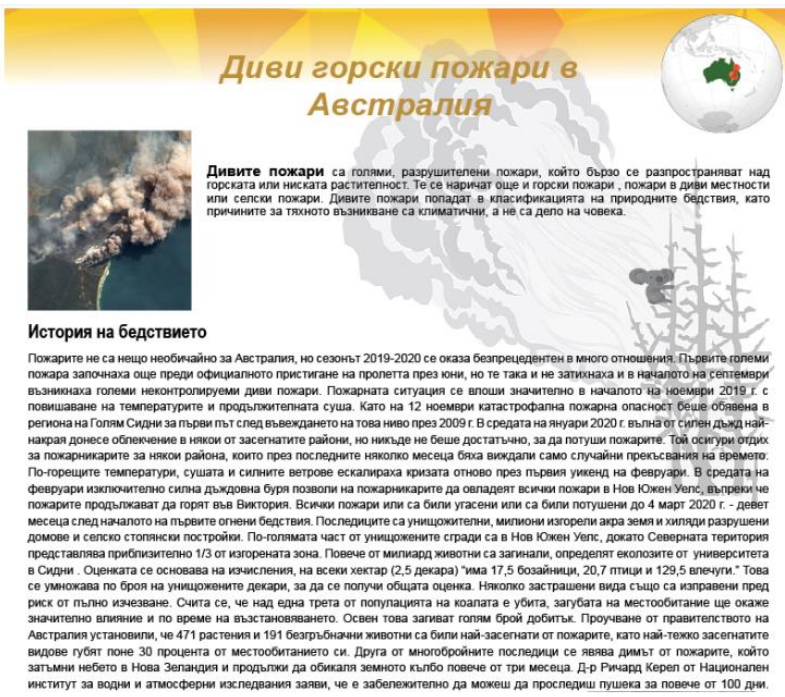

Figure 7. Top of the information page

At the bottom of the page are the consequences of the disaster. Two types of statistics are added, one of them presents the effects by numbers, supported by specially selected and coloured symbols (Figure 8.), the purpose of which is to graphically represent the data. And the other data are presented in text, making it easy to understand why these disasters can easily be called significant. And to complete the design, and because it is easiest to present something by showing it visually, are placed real images of the consequences of disasters.

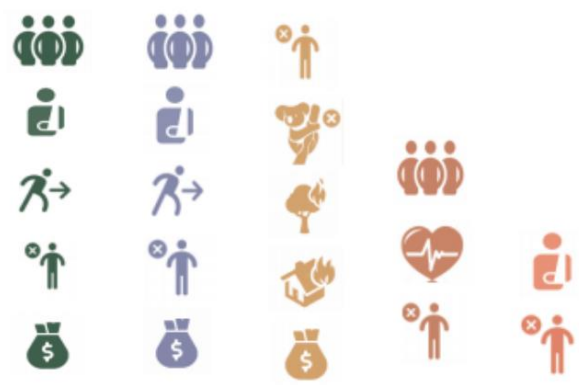

Figure 8. Graphic symbols representing the statistics.

And in combination with the maps, the atlas turns out colourful and convenient (Figure 9).
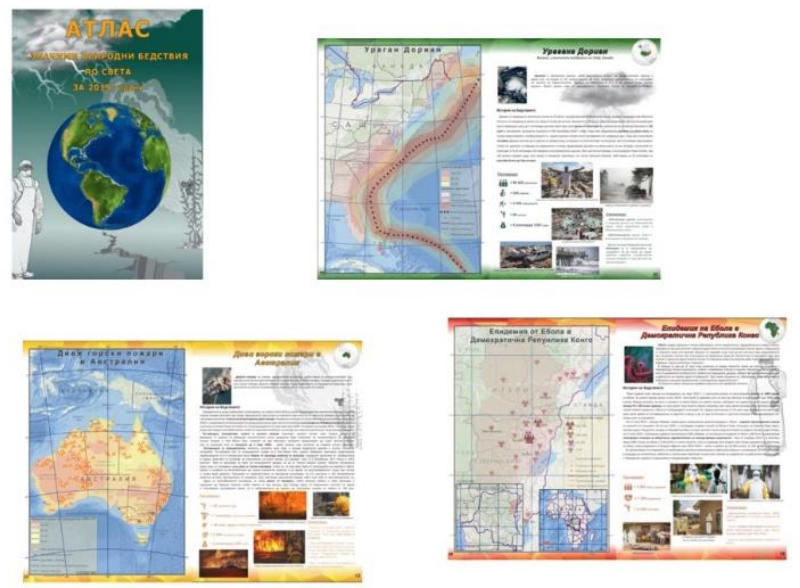

Figure 9. Pages of the "Atlas of significant natural disasters, around the world, for 2019".

\section{Conclusions}

The main focus in this paper is on showing the benefits of atlas mapping for natural disasters, and the design of "Atlas of significant natural disasters, around the world, for 2019". We believe that by collecting and providing information for disasters presented by maps, in such an accessible way, can be achieved a universal increase in the level of awareness of the natural disasters. It is important that all the available information for the disasters, be collected and made easy to understand by everyone. This will increase the percentage of people which will be prepared and can react properly in a disaster situation and the human and financial losses can be reduce. The created atlas is one example, it is global but can easily be made only for one a country, region or city, but its purpose and design will be preserved.

\section{References}

Amandeep Kaur, Sandeep K. Sood, 2019. Ten years of disaster management and use of ICT: a scientometric analysis, Earth Science Informatics, Issue 1/2020

Bandrovaa, T., Zlatanovab, S., Konecny, M., 2012. ThreeDimensional Maps For Disaster Management, ISPRS Annals of the Photogrammetry, Remote Sensing and Spatial Information Sciences, Volume I-4, 2012 XXII 
ISPRS Congress, 25 August - 01 September 2012, Melbourne, Australia p 246

Centre for Research on the Epidemiology of Disasters (CRED), 2020. Natural Disasters 2019 Analysis, accessed on 5 Aug 2020

EM-DAT, 2021. The International Disaster Database, Centre for Research on the Epidemiology of Disasters CRED, General Classification, accessed on 15 May 2021

Konecny M, Zlatanova S, Bandrova T, 2010. Geographic Information and Cartography for Risk and Crisis Management. Towards Better Solutions. Berlin Heidelberg: Springer, p 446

National Research Council, 2007. Successful Response Starts with a Map: Improving Geospatial Support for Disaster Management. Washington, DC: The National Academies Press, p 198, doi:10.17226/11793

Zlatanova S, Li J, 2008. Geospatial Information Technology for Emergency Response. Taylor \& Francis Group, London, UK, p 394 